\title{
A biophysical model for transcription factories
}

\author{
Ana Z Canals-Hamann ${ }^{1}$, Ricardo Pires das Neves ${ }^{1}$, Joyce E Reittie ${ }^{1}$, Carlos Iñiguez ${ }^{2}$, Shamit Soneji ${ }^{1}$, Tariq Enver ${ }^{1}$, \\ Veronica J Buckle ${ }^{1}$ and Francisco J Iborra ${ }^{1,3^{*}}$
}

\begin{abstract}
Summary
Transcription factories are nuclear domains where gene transcription takes place although the molecular basis for their formation and maintenance are unknown. In this study, we explored how the properties of chromatin as a polymer may contribute to the structure of transcription factories. We found that transcriptional active chromatin contains modifications like histone $\mathrm{H} 4$ acetylated at Lysine 16 (H4K16ac). Single fibre analysis showed that this modification spans the entire body of the gene. Furthermore, H4K16ac genes cluster in regions up to $500 \mathrm{~Kb}$ alternating active and inactive chromatin. The introduction of H4K16ac in chromatin induces stiffness in the chromatin fibre. The result of this change in flexibility is that chromatin could behave like a multi-block copolymer with repetitions of stiff-flexible (active-inactive chromatin) components. Copolymers with such structure self-organize through spontaneous phase separation into microdomains. Consistent with such model H4K16ac chromatin form foci that associates with nascent transcripts. We propose that transcription factories are the result of the spontaneous concentration of H4K16ac chromatin that are in proximity, mainly in cis.
\end{abstract}

Keywords: Epigenetics, Biophysics, H4K16Ac, BrUTP, Transcription Factories, RNA pol II, Nuclear organization

\section{Background}

Transcription in eukaryotes is organized in transcription factories (TFs), which are nuclear domains where several genes are grouped to be transcribed together [1,2] [3-5]. The current opinion is that the genes in a TF interact by a looping mechanism [5-8]. It has been suggested that chromatin looping plays an important role in controlling gene activity by bringing together promoters and enhancers or TFs [9]. Some studies suggest that promoterenhancer loops are maintained by the interaction of proteins associated with these cis-regulatory elements [10]. This interaction precedes chromatin activation, which is required for gene relocation to the TF by an unknown mechanism [11]. It has been proposed that TFs are maintained by depletion attraction forces (excluding volume effect) between RNA pol II molecules [12]. However, experimental evidence has shown that genes remain at the factory even when active RNA pol II is not present [13]. This makes the excluding volume model very improbable and suggests that this structure is not the result of

\footnotetext{
* Correspondence: fjiborra@cnb.csci.es

${ }^{1}$ MRC Molecular Haematology Unit, Weatherall Institute of Molecular Medicine, John Radcliffe Hospital, Headington, Oxford OX3 9DS, UK ${ }^{3}$ Departamento de Biología Molecular y Celular, Centro Nacional de Biotecnología, CSIC, Darwin 3, Campus de Canto Blanco, Madrid 28049, Spain Full list of author information is available at the end of the article
}

transcription. Instead experimental evidence points to histone acetylation as being responsible for loop formation [11]. For these reasons we explored the possible contribution of chromatin acetylation in the formation of TFs.

Chromatin at the TF is decondensed [2] and contains active transcription marks like histone acetylation or H3K36me3 [14]. Among all the possible Lysine residues that can be acetylated, H4K16Ac is very special because it prevents the formation of compacted chromatin by inhibiting the inter-fibre interaction [15-18]. Moreover, H4K16 acetylation is associated with both active chromatin [19] and with the active transcription marker H3K4me3 [19-21].

\section{Results and discussion}

To confirm whether H4K16Ac is associated with active chromatin, we analysed the distribution of H4K16Ac in the nucleus of the TFs of circulating lymphocytes. The TFs were visualised as sites of incorporation of Br-UTP into nascent RNA. TFs appeared as discrete foci distributed along the edge of condensed chromatin (Figure 1a) as previously described in other cell types [2,22,23]. H4K16Ac was scattered in foci overlapping or very close to these Br-RNA sites (Figure 1a). To study the extent and degree of the hyper-acetylated chromatin in

\section{() Biomed Central}



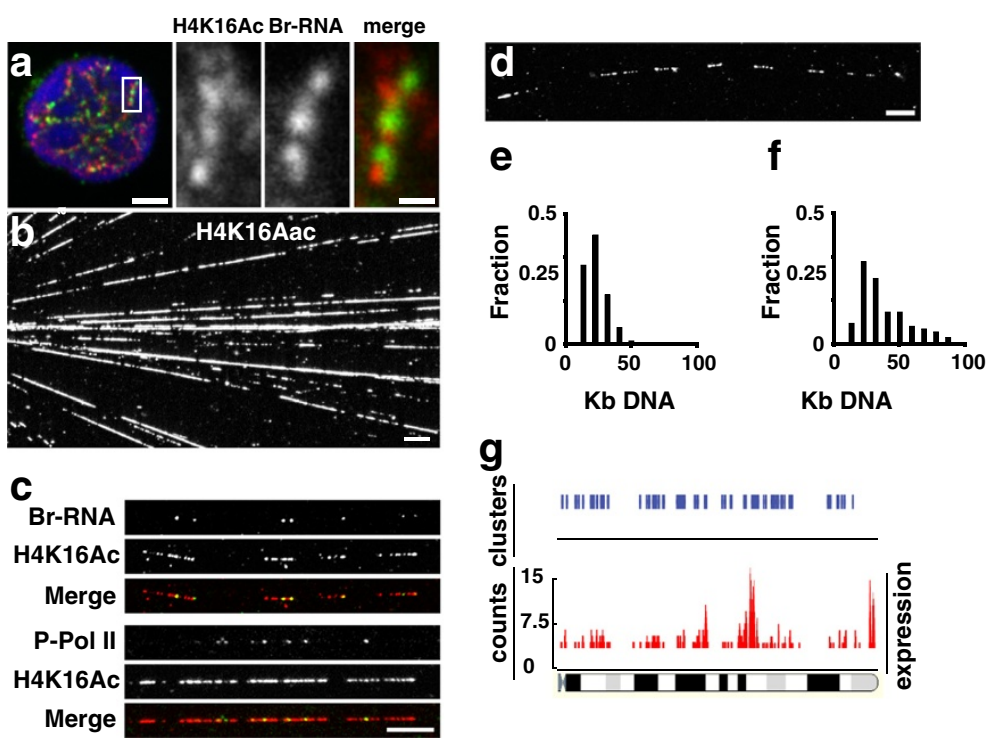

Figure 1 Transcription on acetylated chromatin. (a) The nascent Br-RNA (green) and H4 K16Ac (red) signals are closely associated. BrUTP incorporation in human lymphocyte was carried out for 15 min and after fixation immunolabeled together with histone H4 K16Ac (rabbit antibody). (b) The deconstruction of cell nuclei. After sarkosyl treatment, chromatin was spread and immunolabelled with H4 K16ac, to show tracks of hyperacetylated chromatin. (c) The colocalisation of Br-RNA after BrUTP and H4 K16ac. Br-RNA appears as little spots on tracks of acetylated chromatin, equivalent images were obtained when P-RNA pol II (Ser ${ }^{2}$ ) antibody (H5) was used. (d) Tracks of acetylated chromatin appeared in clusters. (e) The distribution of sizes of chromatin acetylated tracks. (f) The distribution of sizes of chromatin between consecutive acetylated tracks. (g) Expression data from FCDP mix cells on mouse chromosome 10. Expressed genes tend to cluster along the chromosome. For cluster analysis we used a $500 \mathrm{~Kb}$ window. When clustering was significant ( $p>0.95$ ) a blue line is drawn. Bars: $a=2 \mu \mathrm{m}$, merge $=200 \mathrm{~nm} ; b, c, d=10 \mu \mathrm{m}$.

individual transcription units (TUs), we deconstructed the nuclei of these cells by making chromatin spreads. Under these conditions active RNA polymerases and epigenetic modifications of the chromatin are preserved. This treatment disassembled nuclei and spread templates over a wide area. The DNA adopts a linear structure with no visible nucleosomes [22] and 95\% of active polymerases remain associated with the DNA [24]. When we stained the chromatin spreads with antibodies against H4K16Ac, they showed almost continuous fluorescent tracks along the DNA fibres (Figure 1b). These H4K16Ac tracks corresponded with active chromatin, as demonstrated by co-localisation of H4K16Ac with the nascent transcripts that were labelled either in vivo or in vitro by using Bromo-Uridine (BrU) or BrUTP respectively. The area covered by acetylated histones was larger than that stained by the nascent transcripts (Figure 1c). This was to be expected because histone acetylation extends over long stretches of genes, whilst only a few RNA pol II molecules are ever found on a given gene [22]. Nevertheless, to demonstrate that the distribution of active RNA pol II molecules is not an artifact of non-natural nucleotide incorporation, we carried out chromatin spreads with cells where transcription was not labelled with BrU. Our experiments demonstrated a similar co-localisation of H4K16Ac with P-RNA pol II (hyper-phosphorylated $\mathrm{Ser}_{2}$ ) (Figure 1c).
The chromatin spreading technique allowed us to measure the length of H4K16Ac tracks. The distribution of H4K16Ac stretches showed a lognormal distribution with average size of $\sim 15 \mathrm{~Kb}$ (Figure 1e). H4K16Ac tracks rarely appeared isolated, instead they tended to cluster, spanning several hundreds of $\mathrm{Kb}(348 \pm 90$; range 235$530 \mathrm{~Kb}$ ) (Figure 1d). The extension of the gaps between two consecutive H4K16Ac tracks in the cluster showed a lognormal distribution with an average distance of $\sim 30$ $\mathrm{Kb}$ (Figure 1f). The analysis of the polymerases loaded onto H4K16Ac tracks showed that not all the tracks were stained with Br-RNA or P-RNA pol II. The number of nascent transcripts or P-RNA pol II per track was low $(0.7 \pm 1$ transcripts/track and $0.8 \pm 0.9$ P-RNA pol II/ track). This was in accordance with our previous findings, suggesting that most of the TUs contain one molecule of RNA pol II [22]. The fact that some H4K16Ac tracks of chromatin were not associated to RNA pol II or Br-RNA could reflect a temporal discrepancy between the transcription and acetylation processes of chromatin. Indeed, transcription by RNA pol II takes only a few minutes [25-27] while deacetylation of active chromatin can take several hours [28], providing a molecular memory of recently-transcribed chromatin. On the other hand, H4K16Ac tracks are not a special feature of lymphocytes as we were able to find the same chromatin organisation in all the mammalian cell types tested 
including: Hela, Epstein Barr transformed lymphocytes, human lymphocytes, primary human fibroblasts, primary mouse fibroblasts and murine erythroleukemia cells (both differentiated and undifferentiated).

The clusters in all the different cell types analysed were identical with respect to the number of TUs $(8 \pm 2$ TUs/Cluster), suggesting that co-linear active genes expressed at the same time, in agreement with the analysis of expression data using FDCP mix cells [29]. The sliding window analysis (applying a window of $500 \mathrm{~Kb}$ ) over the entire genome showed that genes are active in clusters (Figure 1g), in accordance with our chromatin spreads data. Moreover, our results are consistent with the co-expression data after a Serial Analysis of Gene Expression where the cluster size was $<500 \mathrm{~Kb}$ [30]. From these data we can conclude that co-linear TUs are active at the same time in the same cell.

\section{How are these TUs organised in the cell nucleus?}

Collinear active TUs are enriched in H4K16Ac which confers stiffness and inhibits inter-fiber interaction [15-17]. In this way, chromatin appears as a multi-block copolymer
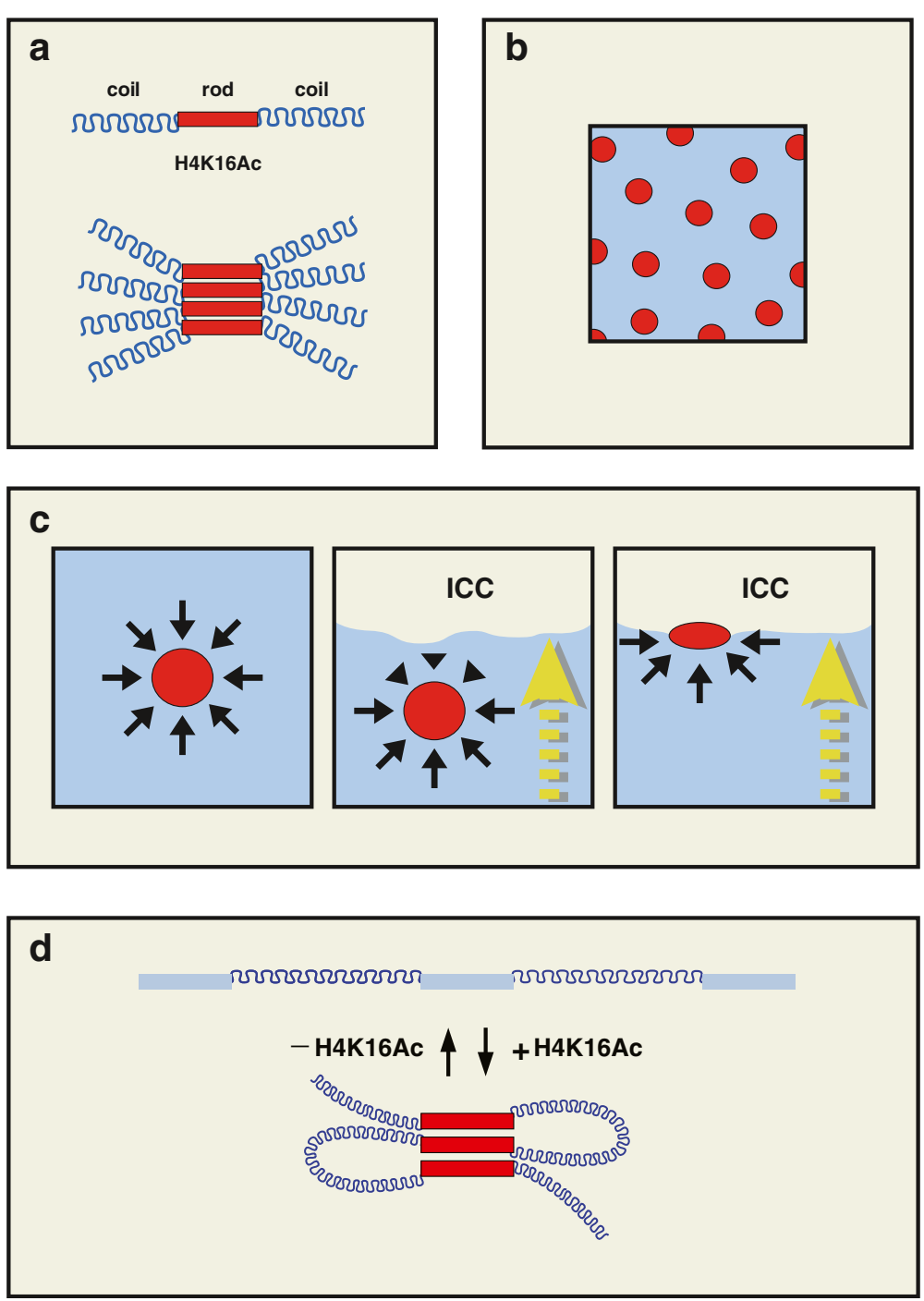

Figure 2 Multi-block copolymer. (a) Chromatin exists as a multi-block copolymer with flexible and stiff (coil-rod) chromatin. Stiff blocks selfinteract creating an anisotropic crystalline phase (pile of red blocks). (b) These segregated blocks generate a symmetric microphase pattern where the stiff phase (minor component) self organise in micro-spheres (red circles) embedded in the major phase of flexible DNA (blue background). (c) The organization of the active chromatin microphase in the cell nucleus. Microspheres remain in a fixed position due to the balance of opposing forces. In the cell nucleus the existence of interphases creates an imbalance between the forces acting in opposing directions, with a net force pushing the microspheres to the interphase. This explains the position of active chromatin at the edge of the condensed chromatin. (d) Model of self-organization of active chromatin. Collinear active gene chromatin is hyper-acetylated which results in its self-interaction with neighbouring TUs. These interactions are destroyed by the deacetylation of chromatin. 
with stiff and flexible monomers (rod-coil) $)_{\mathbf{n}}$ system, where the rod is the stiff active TU. The multi-block copolymers function as amphiphiles whose components segregate into domains to avoid unfavourable contact with each other. In these systems, complete phase separation is prevented by the covalent linkage between the components [31]. The rod block does not have the same conformational entropy as the coil block and this restricts homogeneous packaging. In consequence anisotropic interactions occur between the stiff blocks ending in a liquid crystalline domain where the different TUs are aligned in a high order smectic phase [31] (Figure 2a). Multi-block copolymers can adopt many different structures depending on the relative proportions of the rod and coil phases. For example, when the rod phase is lower than $20 \%$ the structures obtained are microspheres [32] (Figure 2b). A calculation of the amount of active chromatin in a Hela cell line gives a proportion of rods to coils of $\sim 12 \%$, which is consistent with active chromatin separated in many microspheres. These spheres for H4K16Ac chromatin were observed in the cell nucleus of human lymphocytes (the shape factor was 0.93 +0.05 ). Microspheres are regularly distributed in artificial polymers with regular coils and rods resulting from the repulsion forces of coils pushing in all directions. In the cell nucleus TFs are not regularly distributed because the sizes of genes and intergenic distances are not as regular as in artificial polymers. Moreover, H4K16Ac foci concentrated at the edge of condensed chromatin (Figure 1a). A possible reason for this discrepancy is that chromatin cohabits with the inter chromatin compartment (ICC), which is composed by RNPs and proteins. This results in a biphasic system where the ICC (inelastic phase) segregates from chromatin [33], creating an interphase between both components. Under these conditions microspheres containing H4K16Ac may be pushed by the coil polymers to the interphase between chromatin and ICC (Figure 2c), resulting in the localisation along the edge of the chromatin as observed.

A prediction of the multi-block copolymer model is that microphase separation must persist as long as H4K16Ac is present in chromatin. In fact, H4K16Ac foci were unperturbed by treatments like $2 \mathrm{M} \mathrm{NaCl}$ extraction, which disrupts chromatin; transcription inhibition by DRB (5,6-Dichlorobenzimidazole $1-\beta$-D-ribofuranoside), which reduces RNA pol II transcription by $98 \%$; and heat shock $\left(1 \mathrm{~h} 45^{\circ} \mathrm{C}\right)$, which releases RNA pol II from the DNA [26] (Additional file 1: Figure S1). The only way to disrupt these foci was by formamide treatment, which works as a solvent for the electrostatic selfassembled polymers (Additional file 2: Figure S2).

These experiments contradict the excluding volume model [12] and are in agreement with the multi-block microphase separation hypothesis proposed in this study.
The next question about the genes in a TF is where they come from. Several studies have shown that genes in cis and in trans are able to interact in the same TF [5-7]. However, the analysis of chromatin spreads showed that collinear genes are active in the same cell at the same time. This guarantees that several H4K16Ac tracks are in close proximity. Therefore, most of these collinear TUs would probably aggregate in the same microsphere, as occurs in similar situations with the multi-block copolymers [34]. The experimental evidence from chromosome configuration capture analysis suggests that local chromatin is the primary source of interaction for any genomic loci [8]. Nevertheless, we cannot exclude the possibility that some genes located further away in the same chromosome or in another chromosome can interact due to proximity or chromatin folding.

Finally, a remarkable feature of TFs is their constant size across species and differentiation stages [23]. According to the multi-block copolymer model for chromatin organisation, the way to change the size of H4K16Ac foci (and consequently TFs) is by increasing the number of active genes in a given region or by unrestricting the mobility of the active chromatin. The latter has been reported in experiments using plasmids that rendered larger TFs than the endogenous ones $[35,36]$.

In summary, we present evidence of the relationship between epigenetic marks and the TF structure. Our model proposes that active chromatin self-organises in the nucleus due to the special physical properties of H4K16Ac modified chromatin. Therefore, our model implies that chromatin becomes activated (H4K16Ac modified) before joining a TF. This is conceptually very different from current transcription factory model, which proposes that genes are targeted to TFs to "enhance production by concentrating the relevant machines, resources, and expertise in one place" [37].

\section{Materials and methods}

\section{Transcription in vivo and in vitro}

For in vivo transcription, cells grown on coverslips were incubated in presence of $2.5 \mathrm{mM} \mathrm{BrU}$ for several min.

For in vitro transcription, cells grown on coverslips were treated as described [3].

\section{Chromatin spreading}

Cells $\left(10^{3}\right.$ cells in $\left.5 \mu \mathrm{l}\right)$ were spotted onto a $22 \times 50 \mathrm{~mm}$ glass slide and $5 \mu \mathrm{l}$ of lyses buffer were added (Lyses buffer: $1 \%$ sarkosyl, $25 \mathrm{U} / \mathrm{ml}$ ribonuclease inhibitor, $10 \mathrm{mM}$ EDTA, and $100 \mathrm{mM}$ Tris-HCl (pH 7.4)). After $10 \mathrm{~min}$ at $20^{\circ} \mathrm{C}$, the slide was tilted to allow the drop to run down. Samples were air-dried and fixed in $4 \%$ Paraformaldehyde for $10 \mathrm{~min}$. Clusters were defined as two o 
more hyper acetylated tracks in less than $100 \mathrm{~Kb}$. For quantification of clusters of hyper acetylated chromatin between 150 and 200 tracks of hyper acetylated chromatin were analysed.

\section{Immunofluorescence}

After blocking for non-specific antibody binding, immunolabelling was carried out as described [3]. For detection of primary transcripts, we used mouse anti-IdU/ BrdU (5 mg/ml; Caltag Laboratories, Burlingame, CA). For detection of H4K16ac we used antibodies raised in rabbit and mouse (Serotec, Kidlington, UK, Abcam). RNA pol II hyperphosphorylated in Ser 2 was detected with H5 antibody (Covance). Secondary antibodies donkey anti-mouse IgG or IgM tagged with Cy3 (1/200 dilution; Jackson ImmunoResearch, Bar Harbor, ME) and donkey anti-rabbit IgG tagged with Alexa 488 (1/200; prepared using a Molecular Probes kit, Inc., Eugene, OR). DNA staining was performed with $200 \mathrm{nM}$ TOPRO-3 (Molecular Probes) for $5 \mathrm{~min}$. Then coverslips were mounted on slides using Vectashield (Vector laboratories), and images were collected using a Radiance 2000 confocal microscope (Bio-Rad Laboratories, Hemel Hempstead, Herts, UK), Distances were measured using EasiVision software (Soft Imaging Systems GmbH, Münster, Germany) and data exported to Excel (Microsoft) for analysis.

The degree of spreading of the chromatin was measured by hybridising the spreads with a fragment of DNA of $47.26 \mathrm{~Kb}$; the spreading was $3.9+0.2 \mathrm{~Kb} / \mu \mathrm{m}$.

\section{Microarrays and sliding window analysis}

Mouse FCDP mix cells were used. cRNA synthesis and hybridisation to oligonucleotide array were performed as described [29].

The sliding window analysis was performed by applying a window of $500 \mathrm{~Kb}$ over the chromosomes and moved at $5 \mathrm{~Kb}$ steps along a chromosome to know whether the genes contained in that window were more likely to be transcribed together than just by chance.

\section{Additional files}

Additional file 1: Figure S1. Stability of $\mathrm{H} 4 \mathrm{~K} 16 \mathrm{Ac}$ foci. Resistance of $\mathrm{H} 4$ K16Ac foci to various treatments that disrupted transcription or chromatin structure. The aspect of $\mathrm{H} 4 \mathrm{~K} 16 \mathrm{Ac}$ foci did not change after DRB treatment (2h $150 \mu \mathrm{M})$ or heat shock $(\mathrm{Hs})$ for $1 \mathrm{~h}$ at $45^{\circ} \mathrm{C}$. Both treatments led to the release of RNA pol II from the genes. These foci were also resistant to $\mathrm{NaCl}$ extraction (cells permeabilised with $0.05 \%$ Triton $\mathrm{X} 100$ for $5 \mathrm{~min}$ in PBS at $4^{\circ} \mathrm{C}$ followed by 10 min extraction with $2 \mathrm{M} \mathrm{NaCl}$ for $10 \mathrm{~min}$ ). The images were pseudo-coloured for display. The bottom bar shows the scale of pseudo-colours used.

Additional file 2: Figure S2. H4 K16Ac foci are disassembled by formamide. The resistance of $\mathrm{H} 4 \mathrm{~K} 16 \mathrm{Ac}$ foci to formamide treatment. Cells were incubated for $5 \mathrm{~min}$ in PBS with different concentrations of formamide $(0,25,50$ and $100 \%)$ then fixed with $4 \%$ paraformaldehyde and immunolabelled with H4K16Ac antibodies. (a) The H4K16Ac foci were disassembled by formamide treatment, as can be seen from the change in the staining pattern, which is more diffuse and less intense than the control. The images were pseudo-coloured for display. (b) The deconstruction of the foci was quantified by the change in the pixel intensity variation coefficient (SD/mean). This analysis was performed by measuring the mean intensity and the standard deviation (SD) of the H4K16Ac signal of the nuclear areas in at least 200 cells for each treatment. The images were pseudo-coloured for display. The bottom bar shows the scale of pseudo-colours used.

\section{Authors' contributions}

AZC-H, RPN and JER performed some of the immunocytochemical experiments and acquired data. SS and TE, performed the transcriptomic and the statistical analyses. Cl participated in the draft the manuscript. VJB was in charge of the DNA fish experiments \& FJ conceived of the study, and participated in its design and coordination and helped to draft the manuscript. All authors read and approved the final manuscript.

\section{Acknowledgements}

The authors would like to thank Elspeth McFarlane for helping with the English. To: Ministerio de Economia y Competitividad, (Spain) (Grant number: BFU2009-10792) and The Medical Research Council (UK), for supporting this work. We also thank Fundação Ciência e Tecnologia, Portugal for funding RPN.

\section{Author details}

${ }^{1}$ MRC Molecular Haematology Unit, Weatherall Institute of Molecular Medicine, John Radcliffe Hospital, Headington, Oxford OX3 9DS, UK. Departamento de Biotecnología, Universidad de Alicante, Alicante 03080, Spain. ${ }^{3}$ Departamento de Biología Molecular y Celular, Centro Nacional de Biotecnología, CSIC, Darwin 3, Campus de Canto Blanco, Madrid 28049, Spain.

Received: 14 August 2012 Accepted: 5 December 2012 Published: 9 February 2013

\section{References}

1. Iborra FJ, Jackson DA, Cook PR: The path of transcripts from extranucleolar synthetic sites to nuclear pores: transcripts in transit are concentrated in discrete structures containing SR proteins. Journal of cell science 1998, 111:2269-2282.

2. Iborra FJ, Pombo A, Jackson DA, Cook PR: Active RNA polymerases are localized within discrete transcription "factories' in human nuclei. Journal of cell science 1996, 109:1427-1436.

3. Iborra FJ, Escargueil AE, Kwek KY, Akoulitchev A, Cook PR: Molecular crosstalk between the transcription, translation, and nonsense-mediated decay machineries. Journal of cell science 2004, 117:899-906.

4. Iborra FJ: The path that RNA takes from the nucleus to the cytoplasm: a trip with some surprises. Histochem Cell Biol 2002, 118:95-103.

5. Osborne CS, Chakalova L, Brown KE, Carter D, Horton A, Debrand E, Goyenechea B, Mitchell JA, Lopes S, Reik W, Fraser P: Active genes dynamically colocalize to shared sites of ongoing transcription. Nat Genet 2004, 36:1065-1071.

6. Osborne CS, Chakalova L, Mitchell JA, Horton A, Wood AL, Bolland DJ, Corcoran $A E$, Fraser $P$ : Myc dynamically and preferentially relocates to a transcription factory occupied by Igh. PLOS Biol 2007, 5:e192.

7. Chakalova L, Carter D, Debrand E, Goyenechea B, Horton A, Miles J, Osborne C, Fraser P: Developmental regulation of the beta-globin gene locus. Prog Mol Subcell Biol 2005, 38:183-206.

8. Simonis M, Klous $P$, Splinter E, Moshkin $Y$, Willemsen $R$, de Wit E, van Steensel $B$, de Laat W: Nuclear organization of active and inactive chromatin domains uncovered by chromosome conformation captureon-chip (4C). Nat Genet 2006, 38:1348-1354.

9. Chambeyron S, Bickmore WA: Does looping and clustering in the nucleus regulate gene expression? Curr Opin Cell Biol 2004, 16:256-262.

10. Kim SI, Bultman SJ, Kiefer CM, Dean A, Bresnick EH: BRG1 Requirement for long-range interaction of a locus control region with a downstream promoter. Proc Natl Acad Sci U S A 2009, 106:2259-2264. 
11. Kim A, Dean A: Chromatin loop formation in the beta-globin locus and its role in globin gene transcription. Mol Cells 2012.

12. Marenduzzo D, Micheletti C, Cook PR: Entropy-driven genome organization. Biophys J 2006, 90:3712-3721.

13. Mitchell JA, Fraser P: Transcription factories are nuclear subcompartments that remain in the absence of transcription. Genes Dev 2008, 22:20-25.

14. Muller WG, Rieder D, Karpova TS, John S, Trajanoski Z, McNally JG: Organization of chromatin and histone modifications at a transcription site. J Cell Biol 2007, 177:957-967.

15. Shogren-Knaak M, Ishii H, Sun JM, Pazin MJ, Davie JR, Peterson CL: Histone H4-K16 acetylation controls chromatin structure and protein interactions. Science 2006, 311:844-847.

16. Braunstein M, Sobel RE, Allis CD, Turner BM, Broach JR: Efficient transcriptional silencing in saccharomyces cerevisiae requires a heterochromatin histone acetylation pattern. Mol Cell Biol 1996, 16:4349-4356.

17. Grunstein M: Histone acetylation in chromatin structure and transcription. Nature 1997, 389:349-352.

18. Robinson PJ, An W, Routh A, Martino F, Chapman L, Roeder RG, Rhodes D: $30 \mathrm{~nm}$ chromatin fibre decompaction requires both H4-K16 acetylation and linker histone eviction. J Mol Biol 2008, 381:816-825.

19. Wang Z, Zang C, Rosenfeld JA, Schones DE, Barski A, Cuddapah S, Cui K, Roh TY, Peng W, Zhang MQ, Zhao K: Combinatorial patterns of histone acetylations and methylations in the human genome. Nat Genet 2008 40:897-903.

20. Millar CB, Grunstein M: Genome-wide patterns of histone modifications in yeast. Nat Rev Mol Cell Biol 2006, 7:657-666

21. O'Neill LP, VerMilyea MD, Turner BM: Epigenetic characterization of the early embryo with a chromatin immunoprecipitation protocol applicable to small cell populations. Nat Genet 2006, 38:835-841.

22. Jackson DA, Iborra FJ, Manders EM, Cook PR: Numbers and organization of RNA polymerases, nascent transcripts, and transcription units in HeLa nuclei. Molecular biology of the cell 1998, 9:1523-1536.

23. Faro-Trindade I, Cook PR: A conserved organization of transcription during embryonic stem cell differentiation and in cells with high $C$ value. Mol Biol Cell 2006, 17:2910-2920.

24. Hawley DK, Roeder RG: Functional steps in transcription initiation and reinitiation from the major late promoter in a HeLa nuclear extract. J Biol Chem 1987, 262:3452-3461.

25. Kimura H, Sugaya K, Cook PR: The transcription cycle of RNA polymerase II in living cells. The Journal of cell biology 2002, 159:777-782.

26. Hieda M, Winstanley H, Maini P, Iborra FJ, Cook PR: Different populations of RNA polymerase II in living mammalian cells. Chromosome research: an international journal on the molecular, supramolecular and evolutionary aspects of chromosome biology 2005, 13:135-144.

27. das Neves RP, Jones NS, Andreu L, Gupta R, Enver T, Iborra FJ: Connecting variability in global transcription rate to mitochondrial variability. PLOS Biol 2010, 8:e1000560.

28. Kouskouti A, Talianidis I: Histone modifications defining active genes persist after transcriptional and mitotic inactivation. EMBO J 2005, 24:347-357.

29. Bruno L, Hoffmann R, McBlane F, Brown J, Gupta R, Joshi C, Pearson S, Seidl T, Heyworth C, Enver T: Molecular signatures of self-renewal, differentiation, and lineage choice in multipotential hemopoietic progenitor cells in vitro. Mol Cell Biol 2004, 24:741-756.

30. Lercher MJ, Urrutia AO, Hurst LD: Clustering of housekeeping genes provides a unified model of gene order in the human genome. Nat Genet 2002, 31:180-183.

31. Olsen BDSR: Self-assembly of rod-coil block copolymers. Materials Science and Engineering $R$ 2008, 62:37-66.

32. Matsen MW, Bates FS: Unifying weak- and strong-segregation block copolymer theories. Macromolecules 1996, 29:1091-1098

33. Iborra FJ: Can visco-elastic phase separation, macromolecular crowding and colloidal physics explain nuclear organisation? Theor Biol Med Model 2007, 4:15.

34. Muthukumar M, Ober CK, Thomas EL: Competing interactions and levels of ordering in self-organizing polymeric materials. Science 1997, 277:1225-1232.
35. Binnie A, Castelo-Branco P, Monks J, Proudfoot NJ: Homologous gene sequences mediate transcription-domain formation. J Cell Sci 2006, 119:3876-3887.

36. Xu M, Cook PR: Similar active genes cluster in specialized transcription factories. J Cell Biol 2008, 181:615-623.

37. Papantonis A, Cook PR: Fixing the model for transcription: the DNA moves, not the polymerase. Transcription 2011, 2:41-44.

doi:10.1186/2046-1682-6-2

Cite this article as: Canals-Hamann et al: A biophysical model for transcription factories. BMC Biophysics 2013 6:2

\section{Submit your next manuscript to BioMed Central and take full advantage of:}

- Convenient online submission

- Thorough peer review

- No space constraints or color figure charges

- Immediate publication on acceptance

- Inclusion in PubMed, CAS, Scopus and Google Scholar

- Research which is freely available for redistribution

Submit your manuscript at www.biomedcentral.com/submit
C Biomed Central 